Int. J. Morphol.,

31(1):177-183, 2013.

\title{
Antropometría del Martillo en Humanos: Un Estudio Anatómico Directo
}

\author{
Anthropometry of the Malleus in Humans: \\ A Direct Anatomic Study
}

Ramirez, L. M.* \& Ballesteros, L. E.**

RAMIREZ, L. M. \& BALLESTEROS, L. E. Antropometría del martillo en humanos: Un estudio anatómico directo. Int. J. Morphol., 31(1):177-183, 2013.

RESUMEN: El conocimiento morfométrico de las estructuras del oído medio, incluidos cada uno de los oscículos, es importante para la comprensión de su complejidad biomecánica. Las características estructurales y dimensionales del martillo son registradas en 23 martillos de población mestiza adulta Colombiana. Las medidas registradas involucran el área de la cabeza, el cuello, el manubrio, el proceso anterior y lateral y el área de la articulación incudo-maleolar. Tres de los diecinueve registros tomados en este estudio pudieron ser comparados concienzudamente con otros estudios que mostraron similitudes. El largo total del martillo fue de 8,18 mm DE 0,24, la longitud del manubrio fue de 4,91 mm DE 0,25 y la proporción del largo del manubrio y el largo total fue de 60,11\% DE 3,47. Conforme a la población estudiada no se logro encontrar variación individual o pareada en la anatomía del martillo lo que lo hace un hueso morfométricamente uniforme y estable.

PALABRAS CLAVE: Oscículos; Martillo; Manubrio; Antropometría; Anatomía.

\section{INTRODUCCIÓN}

Los oscículos (martillo, yunque y parcialmente el estribo) se forman por osificación endocondral de la parte dorsal del primer arco branquial (cartílago de Meckel) (Whyte et al., 2003). El segundo arco branquial (cartílago de Reichert) finaliza lo iniciado por el primer arco branquial en el estribo. Adicionalmente al proceso de osificación endocondral en el martillo, el proceso anterior de éste se forma de células mesenquimales (os goniale), a través de una osificación intramembranosa (Rodriguez-Vazquez et al., 1993). La formación del martillo igualmente está influenciada por la presencia del meato acústico externo (Ishimoto et al., 2004).

La cadena oscicular esta unida a través de articulaciones sinoviales. El martillo se une al yunque y éste a su vez al estribo a través de estas articulaciones sinoviales incudo-maleolar e incudo-estapedial, respectivamente. Estos huesecillos logran romper y ajustar la impedancia acústica que se genera en la energía sonora transmitida de un medio aéreo a uno líquido y en el que sin su presencia, esta energía rebotaría en su gran mayoría. Si la cadena oscicular no estuviera presente más del $90 \%$ de la energía sonora no se transmitiría. De esta forma la mecánica oscicular en la que el martillo inicia su movilidad al reproducir los movimientos timpánicos, convirtiendo el movimiento aéreo de baja densidad en movimientos análogos de mayor densidad en el líquido coclear (Olsewski, 1990).

Es entonces el martillo el que se encarga de recibir de primera mano la energía sonora y a través de su morfología asimétrica, iniciando el apalancamiento oscicular que el sonido origina. La asimetría del martillo se nota al describirlo morfológicamente: tiene una cabeza grande y ovoide en su parte superior, un cuello delgado que contiene dos procesos dispuestos en ángulo recto, uno anterior y uno lateral, para luego y de manera descendente hasta su ápice, el mango o manubrio que esta adherido a la membrana timpánica (formando su ombligo) y una zona flácida (pars flácida) limitada por el proceso lateral con la pars tensa del timpano. La carilla articular de este hueso se ubica en la

\footnotetext{
* Luis Miguel Ramirez - MS, MSc. Profesor Asociado Universidad de Antioquia. Grupo Estomatología Biomédica, Colombia.

** Luis Ernesto Ballesteros - MD, MSc. Profesor Titular Universidad Industrial de Santander. Grupo Variaciones Anatómicas y Biomecánica Tendomuscular, Colombia.
} 
cabeza con dos vertientes divididas por una prominencia media que acopla con la zona media y hendida de la carilla articular del yunque que reproduce la morfología de la carilla maleolar en negativo, formando juntas la articulación incudo-maleolar (Guyton \& Hall, 2007) . Ésta representa en comparación con la carilla incudo-estapedial la más grande de las dos. Desde la perspectiva morfológica y morfométrica se debe matizar el hecho de que estas estructuras óseas son irregulares e imbalanceadas, lo que genera efecto de palanca mecánica en la transmisión sonora debido a los momentos de fuerza que esta morfología genera (Ramirez \& Ballesteros, 2010).

Los primeros registros morfológicos hechos en oscículos datan de mediados del siglo XVI con Andrew Vesalius (Cole, 1949) y aunque valiosos todos los aportes posteriores, la base de datos de estas medidas es más bien insuficiente. Conforme a este realidad, el conjunto de medidas morfométricas del martillo recolectadas en especímenes colombianos genera valor agregado en la escasa base de datos existente pero igualmente puede representar una información valiosa para procedimientos quirúrgicos otológicos de reemplazo protésico, así como su utilidad en modelos matemáticos que estudien el funcionamiento de los oscículos mediante la construcción de modelos virtuales que permitan estudiar la mecánica de la transmisión de la energía sonora.

\section{MATERIAL Y MÉTODO}

Se estudió el martillo mediante micro-disección de bloques de hueso temporal obtenidos de especímenes cadavéricos adultos frescos de raza mestiza, a quienes se les practicó autopsia en el Instituto de Medicina Legal de Bucaramanga (Colombia) con un abordaje superior a través de la fosa craneal media. El receso epitimpánico fue removido con motor y expuesta la cavidad ótica media en donde se luxó el martillo y el yunque en la articulación incudomaleolar y, posteriormente, se desinsertó el matillo de su ligamento anterior, del tendón del músculo del tímpano y por último de la membrana timpánica. Se limpiaron con agua oxigenada durante 2 horas y registraron. La toma de medidas en las muestras de martillo las realizó un solo operador y de manera directa en cada hueso, con calibrador electrónico (Mitutoyo ${ }^{\circledR)}$ ) y microscopía de aumento de $12 x$. Las registros fotográficos fueron logrados con video-microscopio de 50x.

Las zonas medidas en la cabeza y el cuello del martillo (Tabla I y Fig. 1) corresponden al ancho medio-lateral antero-posterior de la cabeza; y al ancho de la carilla articular en sus porciones lateral, medio y medial, así como la longitud medio-lateral de la carilla.

Tabla I. Medidas del martillo en cabeza, cuello y área articular en milímetros.

\begin{tabular}{|c|c|c|c|c|c|c|c|c|c|c|}
\hline $\begin{array}{c}\text { Muestra } \\
1\end{array}$ & $\begin{array}{c}\text { Genero } \\
\mathrm{M}\end{array}$ & $\begin{array}{l}\text { Lado } \\
\text { I }\end{array}$ & $\begin{array}{c}\text { Cabeza M-L } \\
2,76\end{array}$ & $\begin{array}{c}\text { Cabeza A-P } \\
1,72\end{array}$ & $\begin{array}{c}\text { Art Ancho Lat } \\
2,42\end{array}$ & $\begin{array}{c}\text { Art Ancho- } 1 / 2 \\
1,68\end{array}$ & $\begin{array}{c}\text { Art Ancho Medial } \\
2,01\end{array}$ & $\begin{array}{c}\text { Art largo } M-L \\
2,94\end{array}$ & $\begin{array}{c}\text { Cuello A-P } \\
1,69\end{array}$ & $\begin{array}{c}\text { Cuello M-L } \\
0,80\end{array}$ \\
\hline 2 & M & 1 & 2,48 & 1,76 & 1,87 & 1,34 & 1,87 & 2,45 & 1,46 & 0,79 \\
\hline 3 & M & D & 2,85 & 1,92 & 1,53 & 1,49 & 1,74 & 2,74 & 1,53 & 0,61 \\
\hline 4 & M & 1 & 2,72 & 1,88 & 2,33 & 1,82 & 2,16 & 2,72 & 1,41 & 0,85 \\
\hline 5 & $M$ & $\mathrm{D}$ & 2,37 & 1,68 & 1,54 & 1,26 & 1,64 & 2,76 & 1,56 & 0,84 \\
\hline 6 & $\mathrm{~F}$ & $\mathrm{D}$ & 2,56 & 1,86 & 2,24 & 1,31 & 1,5 & 2,72 & 1,68 & 1,08 \\
\hline 7 & $\mathrm{~F}$ & I & 2,42 & 1,84 & 2,02 & 1,55 & 1,52 & 2,28 & 1,4 & 0,74 \\
\hline $\begin{array}{l}8 \\
9\end{array}$ & $M$ & D & $\begin{array}{l}2,47 \\
2,45\end{array}$ & $\begin{array}{l}1,91 \\
191\end{array}$ & $\begin{array}{l}1,47 \\
1,63\end{array}$ & $\begin{array}{l}1,36 \\
152\end{array}$ & $\begin{array}{l}1,55 \\
149\end{array}$ & $\begin{array}{l}2,14 \\
246\end{array}$ & $\begin{array}{l}1,43 \\
140\end{array}$ & $\begin{array}{l}1,08 \\
101\end{array}$ \\
\hline 10 & $\mathrm{M}$ & 1 & 2,61 & $\begin{array}{r}1,74 \\
17\end{array}$ & 1,92 & $\begin{array}{l}1,24 \\
157\end{array}$ & 1,65 & $\begin{array}{l}2,83 \\
2,96\end{array}$ & $\begin{array}{l}1,49 \\
176\end{array}$ & 0,94 \\
\hline 11 & M & D & 2,58 & 1,7 & 1,74 & 1,57 & 1,86 & 2,96 & 1,76 & 0,91 \\
\hline 12 & $M$ & 1 & 2,61 & 1,81 & 1,58 & 1,49 & 1,85 & 2,78 & 1,60 & 1,09 \\
\hline 13 & $M$ & $\mathrm{D}$ & 2,73 & 1,79 & 1,64 & 0,96 & 1,37 & 3,25 & 1,53 & 1,08 \\
\hline 14 & $M$ & D & 2,42 & 1,95 & 2,1 & 1,64 & 1,84 & 2,39 & 1,32 & 0,9 \\
\hline 15 & $M$ & 1 & 2,36 & 1,83 & 2,22 & 1,42 & 1,58 & 2,76 & 1,46 & 1,02 \\
\hline 16 & $M$ & I & 2,37 & 1,76 & 1,99 & 1,98 & 1,45 & 2,29 & 1,45 & 0,85 \\
\hline 17 & $M$ & $D$ & 2,37 & 1,98 & 1,49 & 1,85 & 1,65 & 1,98 & 1,31 & 0,84 \\
\hline 20 & $\mathrm{M}$ & D & 2,55 & 1,77 & 1,92 & 1,47 & 1,56 & 2,75 & 1,90 & 1,08 \\
\hline 21 & $M$ & 1 & 2,54 & 1,76 & 1,79 & 1,68 & 1,50 & 2,89 & 1,79 & 1,15 \\
\hline 22 & $M$ & I & 2,76 & 1,87 & 1,52 & 1,71 & 1,98 & 2,4 & 1,3 & 0,98 \\
\hline 23 & $M$ & D & 2,53 & 1,9 & 2,01 & 2 & 1,64 & 2,25 & 1,4 & 0,95 \\
\hline Prom & & & 2,55 & 1,82 & 1,86 & 1,56 & 1,68 & 2,60 & 1,53 & 0,95 \\
\hline DE & & & 0,15 & 0,09 & 0,29 & 0,26 & 0,20 & 0,30 & 0,17 & 0,14 \\
\hline
\end{tabular}

Cabeza M-L: Ancho medio-lateral de la cabeza. (1 en Fig. 1). Cabeza A-P: Ancho Antero-posterior de la cabeza. (2 en Fig. 1). Art Ancho Lat: Ancho de la carilla articular en su porción lateral. (3 en Fig. 1). Art Ancho- 1/2: Ancho de la carilla articular en su porción media. (4 en Fig. 1). Art Ancho Medial: Ancho de la carilla articular en su porción medial. (5 en Fig. 1). Art largo M-L: Longitud medio-lateral de toda la carilla articular. (6 en Fig. 1). Cuello AP: Ancho antero-posterior del cuello. (7 en Fig. 1). Cuello M-L: Ancho medio-lateral del cuello. (8 en Fig. 1). 

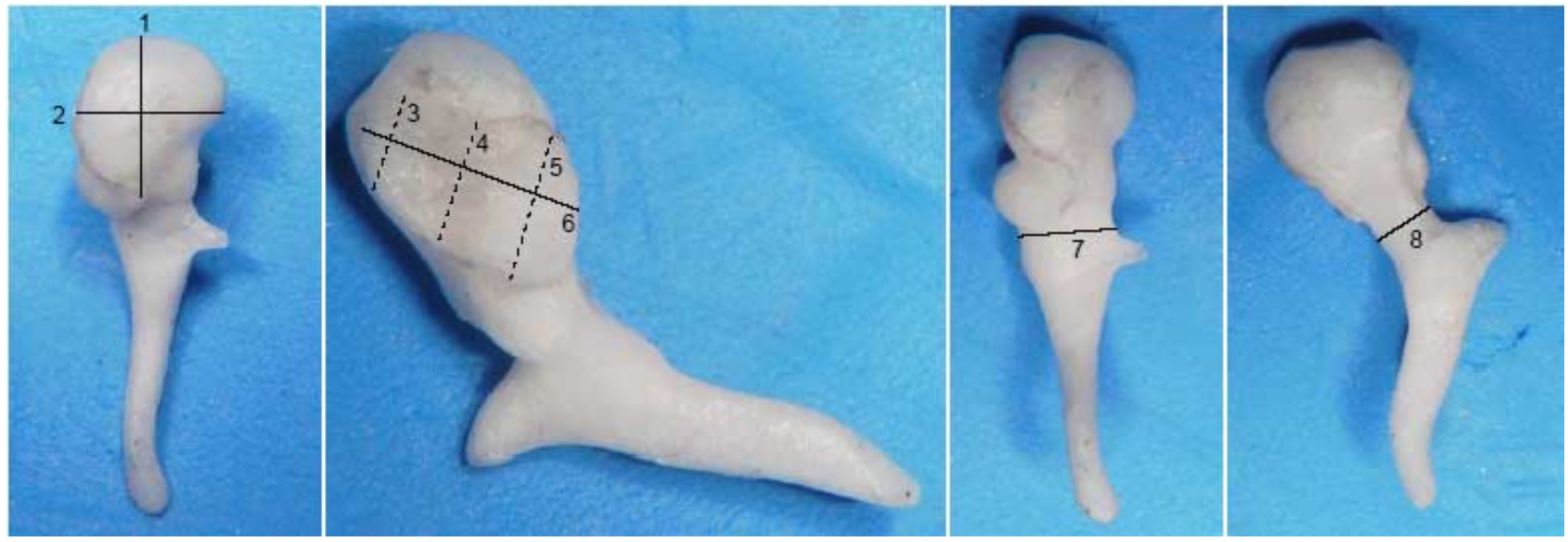

Fig. 1. Medidas de cabeza y cuello del martillo.

Tabla II. Medidas del martillo en proceso anterior-lateral y manubrio en milímetros.

\begin{tabular}{|c|c|c|c|c|c|c|c|}
\hline Muestra & Genero & Lado & Proceso Ant & Proceso Lat & Manubrio-Lat & Manub-Cabeza & Manub-Ant \\
\hline 1 & M & I & 0,81 & 0,85 & 5,32 & 8,45 & 4,76 \\
\hline 2 & M & I & 0,7 & 0,8 & 5,14 & 7,94 & 4,71 \\
\hline 3 & $M$ & D & 0,25 & 0,82 & 4,75 & 8,31 & 4,65 \\
\hline 4 & M & I & 0,28 & 0,74 & 4,81 & 8,19 & 4,69 \\
\hline 5 & $\mathrm{M}$ & D & 0,44 & 0,73 & 5,08 & 8,12 & 4,82 \\
\hline 6 & $\mathrm{~F}$ & D & 0,64 & 0,82 & 4,43 & 8,22 & 4,75 \\
\hline 7 & $\mathrm{~F}$ & I & 0,63 & 0,71 & 4,45 & 8,3 & 4,66 \\
\hline 8 & M & D & 0,55 & 1,07 & 4,83 & 8,38 & 4,91 \\
\hline 9 & M & I & 0,66 & 0,80 & 4,91 & 8,17 & 4,82 \\
\hline 10 & M & I & 0,4 & 0,88 & 4,91 & 8,28 & 4,85 \\
\hline 11 & M & D & 0,22 & 0,96 & 4,53 & 8,39 & 4,73 \\
\hline 12 & $\mathrm{M}$ & I & 1,04 & 1,07 & 5,12 & 8,38 & 4,75 \\
\hline 13 & M & D & 0,85 & 1,04 & 5,10 & 8,44 & 4,98 \\
\hline 14 & $\mathrm{M}$ & D & 0,42 & 1,35 & 4,92 & 7,67 & 4,42 \\
\hline 15 & M & I & 0,69 & 1,23 & 4,74 & 7,68 & 4,38 \\
\hline 16 & $\mathrm{M}$ & 1 & 0,48 & 0,84 & 4,85 & 7,93 & 4,70 \\
\hline 17 & $\mathrm{M}$ & D & 0,31 & 0,99 & 4,86 & 7,83 & 4,44 \\
\hline 18 & M & I & 0,78 & 1,2 & 5,24 & 7,98 & 4,77 \\
\hline 19 & M & D & 0,74 & 1,43 & 4,8 & 8,45 & 4,96 \\
\hline 20 & $\mathrm{M}$ & D & 0,83 & 1,44 & 4,68 & 8,07 & 4,59 \\
\hline 21 & $M$ & I & 0,70 & 1,23 & 5,15 & 8,21 & 4,96 \\
\hline 22 & $\mathrm{M}$ & 1 & 0,58 & 1,04 & 5,25 & 8,2 & 4,8 \\
\hline 23 & $\mathrm{M}$ & D & 0,62 & 0,93 & 5,09 & 8,49 & 5,05 \\
\hline Prom & & & 0,59 & 1,00 & 4,91 & 8,18 & 4,75 \\
\hline DE & & & 0,21 & 0,22 & 0,25 & 0,24 & 0,17 \\
\hline
\end{tabular}

Proceso Ant: Longitud del proceso anterior desde el cuello. (1 en Fig. 2). Proceso Lat: Longitud del proceso lateral desde el eje mayor del cuello. (2 en Fig. 2). Manubrio-Lat: Longitud de ápice del manubrio hasta el proceso lateral. (3 en Fig. 2). Manub-Cabeza: Longitud de ápice del manubrio hasta la zona más rostral de la cabeza. (4 en Fig. 2). Manub-Ant: Longitud de ápice del manubrio hasta el extremo del proceso anterior. (5 en Fig. 2)

En la zona del cuello se registró el ancho antero-posterior y medio-lateral de este, así como los procesos lateral y anterior registrados desde su unión al cuello (Tabla II y Fig. 2). Igualmente y cercano a estos reparos se dividió el registro de la longitud del manubrio en varias medidas desde su ápice hasta tres zonas terminales diferentes que correspondieron el proceso lateral, a la zona más rostral de la cabeza del martillo y el extremo del proceso anterior (Tabla 

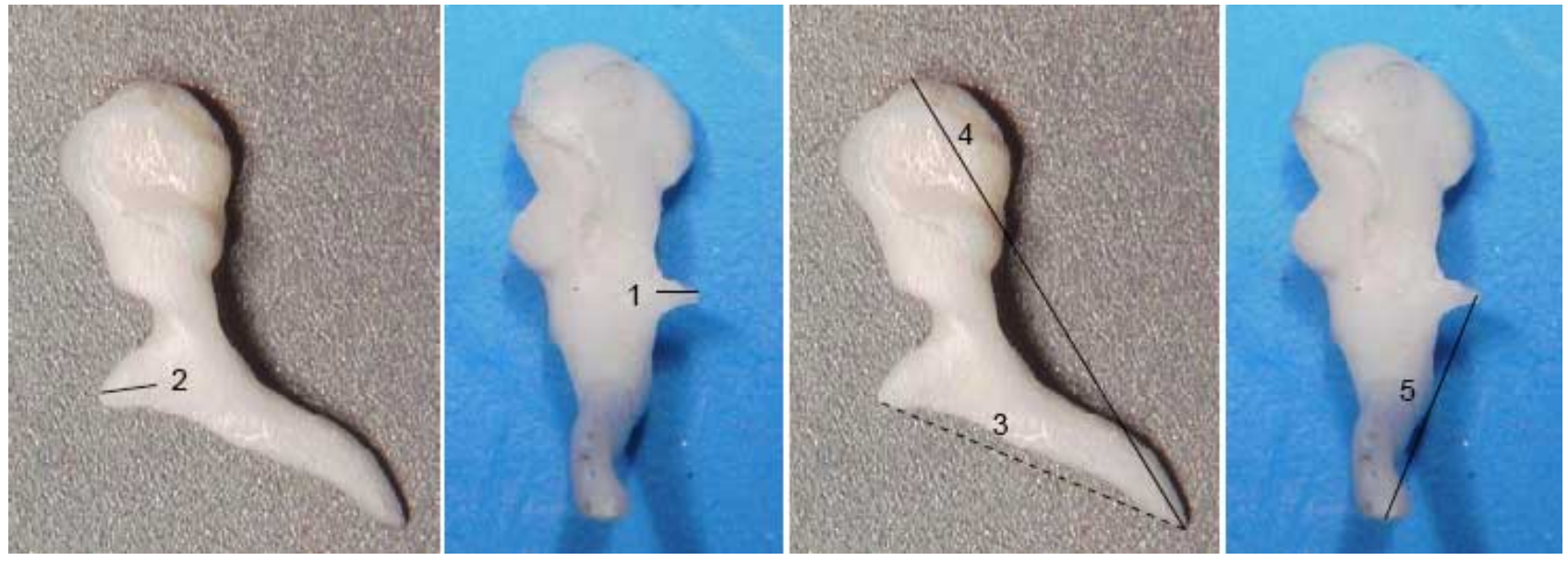

Fig. 2. Longitud del manubrio y de los procesos lateral y anterior del martillo

II y Fig. 2). El ancho y grosor del manubrio se tomó en tres porciones rostro-caudales: superior, media e inferior (Tabla III y Fig. 3).

Se realizaron medidas paramétricas (promedio y desviación estándar) de cada variable y una prueba T pareada en 22 de los especímenes que correspondieron a muestras izquierdas y derechas de un mismo individuo.

\section{RESULTADOS}

En las Tablas I, II y III, se encuentran las medidas pormenorizadas para cada registro de los veintitrés especímenes de martillo estudiadas, con sus promedios y dispersiones individuales. Correspondientes con las tablas, las figuras 1, 2 y 3 muestran los sitios para cada registro.

Tabla III. Medidas del ancho y grosor del manubrio del martillo en milímetros.

\begin{tabular}{|c|c|c|c|c|c|c|c|c|}
\hline Muestra & Genero & Lado & Ancho (R-C) Man Rost & Ancho (R-C) Man 1/2 & Ancho (R-C) Man Caud & Grosor (A-P) Man Rost & Grosor (A-P) Man 1/2 & Grosor (A-P) Man Caud \\
\hline 1 & M & I & 0,95 & 1,25 & 0,73 & 0,79 & 0,71 & 0,61 \\
\hline 2 & $\mathrm{M}$ & 1 & 1,17 & 1 & 0,75 & 0,77 & 0,75 & 0,64 \\
\hline 3 & M & D & 0,98 & 0,97 & 0,64 & 0,86 & 0,68 & 0,66 \\
\hline 4 & M & I & 0,92 & 0,99 & 0,71 & 0,71 & 0,67 & 0,67 \\
\hline 5 & M & $\mathrm{D}$ & 1,11 & 1,11 & 0,79 & 0,77 & 0,63 & 0,54 \\
\hline 6 & $\mathrm{~F}$ & D & 1,29 & 0,93 & 0,67 & 0,87 & 0,8 & 0,58 \\
\hline 7 & $\mathrm{~F}$ & 1 & 0,77 & 0,65 & 0,64 & 1,01 & 0,84 & 0,59 \\
\hline 8 & M & D & 1,05 & 1,06 & 0,65 & 0,89 & 0,75 & 0,70 \\
\hline 9 & M & I & 0,76 & 1,08 & 0,55 & 0,77 & 0,70 & 0,70 \\
\hline 10 & M & 1 & 1,13 & 0,84 & 0,63 & 0,88 & 0,65 & 0,73 \\
\hline 11 & M & D & 1,09 & 0,77 & 0,68 & 0,54 & 0,62 & 0,77 \\
\hline 12 & M & I & 1,21 & 1,04 & 0,76 & 0,96 & 0,66 & 0,70 \\
\hline 13 & $M$ & $D$ & 1,06 & 1,50 & 0,55 & 0,90 & 0,54 & 0,76 \\
\hline 14 & M & $\mathrm{D}$ & 1,11 & 1,04 & 0,75 & 0,66 & 0,65 & 0,49 \\
\hline 15 & M & 1 & 0,88 & 1,08 & 0,67 & 0,77 & 0,63 & 0,43 \\
\hline 16 & $M$ & I & 1,34 & 1,01 & 0,67 & 0,91 & 0,70 & 0,79 \\
\hline 17 & M & D & 1,07 & 1,15 & 0,70 & 0,87 & 0,78 & 0,70 \\
\hline 18 & $M$ & I & 1,08 & 0,97 & 0,54 & 0,88 & 0,63 & 0,66 \\
\hline 19 & $M$ & D & 1,22 & 1,12 & 0,67 & 0,82 & 0,56 & 0,75 \\
\hline 20 & M & $D$ & 1,03 & 1,21 & 0,78 & 1,00 & 0,59 & 0,62 \\
\hline 21 & $M$ & 1 & 1,09 & 1,17 & 0,64 & 0,94 & 0,90 & 0,81 \\
\hline 22 & $M$ & 1 & 1,12 & 1,1 & 0,6 & 0,85 & 0,68 & 0,72 \\
\hline 23 & $M$ & $D$ & 1,24 & 1,17 & 0,64 & 0,83 & 0,61 & 0,73 \\
\hline Prom & & & 1,07 & 1,05 & 0,67 & 0,84 & 0,68 & 0,67 \\
\hline DE & & & 0,15 & 0,17 & 0,07 & 0,11 & 0,09 & 0,10 \\
\hline
\end{tabular}

Ancho (R-C) Man Rost: Ancho (rostro-caudal) del manubrio, porción más superior (proceso lateral). (1 en Fig. 3). Ancho (R-C) Man 1/2: Ancho (rostro-caudal) del manubrio en su porción media. (2 en Fig. 3). Ancho (R-C) Man Caud: Ancho (rostro-caudal) del manubrio en su porción más inferior. (3 en Fig. 3). Grosor (A-P) Man Rost: Grosor (ant-post) del manubrio en su porción más superior (proceso lateral). (4 en Fig. 3). Grosor (A-P) Man 1/2: Grosor (ant-post) del manubrio en su porción media. (5 en Fig. 3). Grosor (A-P) Man Caud: Grosor (ant-post) del manubrio en su porción más inferior. (6 en Fig. 3) 

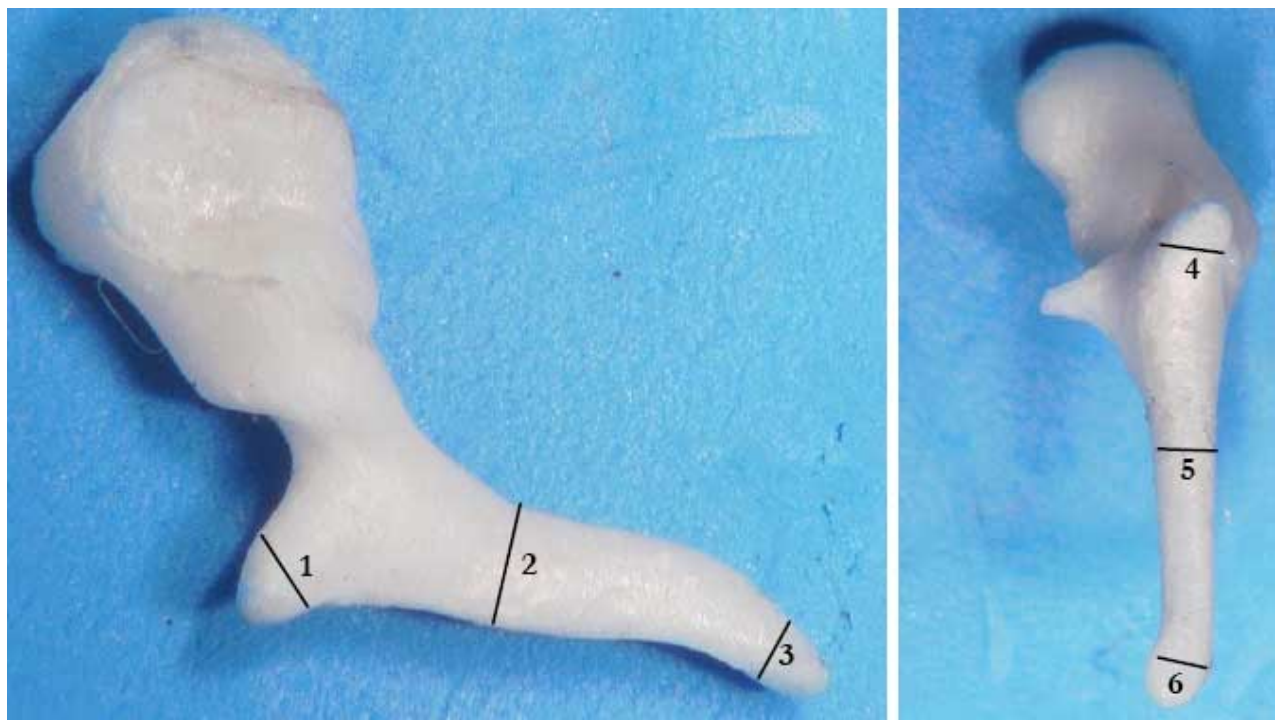

Fig. 3. Ancho y espesor del manubrio del martillo.

\section{DISCUSIÓN}

Se han estudiado tanto la estructura como la función de los huesecillos del oído medio en humanos (Bruintjes, 1990; Anson \& Donaldson 1991). Igualmente la dimensión y el peso de estos comparados entre fetos, adultos y niños que ha mostrado una sutil variación por crecimiento después del nacimiento (Olsewski, 1990). Los patrones de transmisión de fuerzas y su mecánica han sido también estudiados con modelos matemáticos que reconstruyen las formas particulares de los huesecillos para su posterior procesamiento a través de estudios de elemento finito (Tonndorf \& Khanna, 1972; Aycan et al., 1990; Sun et al., 2002, Morris et al., 2004). Parece haber un interés común en la morfología y morfometría de la cadena de oscículos del oído medio en humanos, con fines quirúrgicos, pero también en el diseño y la reconstrucción de implantes electromagnéticos, usando medidas precisas que logran reproducir la transmisión de la energía sonora. (Whyte et al., 2003; Murugasu et al., 2005; Farahani \& Nooranipour, 2008)

De las medidas registradas en este estudio, las longitudes entre cabeza y ápice del manubrio (largo total); y de este último y el proceso lateral (largo del manubrio), así como las proporciones entre estos dos son las únicas medidas comparables con otros estudios (Bouchet \& Giraud, 1968; Malasi, 1968; Harada \& Ishii, 1971; Arensburg et al., 1981; Aycan et al., 1990; Unur et al., 1993, Crevecoeur, 2007; Flohr et al., 2010). Para la longitud de cabeza y ápice de manubrio, el registro de estos investigadores estuvo en un rango entre 7,7 a 8,1 mm que coincide con nuestros registros que fueron en promedio de 8,18 mm DE 0,24. Quam \& Rak (2008), determinaron un rango más amplio de 7,439,31 $\mathrm{mm}$, pero un promedio parecido 8,23 $\mathrm{mm}$ DE 0,41 ; sin embargo sus registros fueron fotográficos y no directos. Harneja \& Chaturvedi (1973) determinaron medidas cercanas inferiores para el largo total de 7,15 mm DE 0,31. Flohr et al. (2010), registraton recientemente esta longitud en 8,11 mm DE 0,37, que se encuentra acorde a la nuestra.

Para la longitud entre cabeza y proceso lateral (largo del manubrio) hubo igualmente similitudes con un promedio de 4,91 mm DE 0,25 en nuestro estudio comparado con la medida registrada por la mayoría de autores (Bouchet \& Giraud, 1968; Malasi, 1968; Harada \& Ishii, 1971; Arensburg et al., 1981; Aycan et al., 1990; Unur et al., 1993, Crevecoeur, 2007; Flohr et al., 2010), que estuvo en un rango entre 4,2 a 4,9 mm, y aunque Quam \& Rak ( 2008) determinaron rangos de 4.22-5.59 mm, sus promedios coinciden con los nuestros (4,94 mm DE 0,31). En el estudio de Flohr et al., ( 2010), el registro de esta longitud fue de 4,91 mm DE 0,34; coincidente a la nuestra. Harneja \& Chaturvedi (1973) mostraron medidas cercanas inferiores para el largo total de 4,22 mm DE 0,35. Como variación se podría contar con el registro de la medida más pequeña de esta distancia con $3,5 \mathrm{~mm}$.

La proporción en porcentaje que ocupa la distancia de cabeza a proceso lateral (largo del manubrio) con el total 
de la distancia entre cabeza a ápice de manubrio (largo total) coincide con nuestros resultados $(60,11 \% \mathrm{DE} 3,47)$ al ser reportada en $61 \%$ (Unur et al., 1993), 56,6\% (Arensburg \& Harell, 1981), 58,3 \% (Crevecoeur, 2007) y 59,97 \% DE 2,81 (Quam \& Rak, 2008).

El estudio de Crevecoeur (2007) permite la comparación del ancho medio-lateral de la cabeza y del ancho antero-posterior de la cabeza en una sola muestra que fue de 2,6 y $2,1 \mathrm{~mm}$, respectivamente y que es similar al promedio en nuestro estudio, para las mismas medidas, de $2,55 \mathrm{~mm}$ DE 0.15 y $1,82 \mathrm{~mm}$ DE 0,09 . Crevecoeur ( 2007) y Quam \& Rak (2008), comparaton el ancho medio-lateral con el promedio del ancho medio-lateral de otros estudios, obteniendo una medida similar a la nuestra con $2,57 \mathrm{~mm}$ DE 0,24 . El estudio de Quam \& Rak (2008), nos permite la comparación del ancho medio-lateral del cuello que fue de $0.99 \mathrm{~mm}$ DE 0,13 y en nuestro estudio de 0,95 mm DE 0,14.

En relación a las variaciones de este hueso, nuestros resultados coinciden con la percepción general, que el martillo es un hueso muy estable sin presencia de importantes variaciones (Sarrat et al., 1992; Unur et al., 1993), incluidos los cambios postnatales (Olsewski, 1990). Arensburg et al., 1981) encontraron los oscículos con morfometría muy estables en poblaciones históricas y prehistóricas con contrastes étnicos, temporales (miles de años) y geográficos amplios. Coincidente, Crevecoeur (2007) estudió la morfometría y la relación filogenética del homínido hombre de diferentes épocas evolutivas midiendo los oscículos desde antes del Neandertal, pasando por el paleolítico y llegando hasta el humano moderno, concluyendo que estos huesecillos son morfométricamente muy estables tanto en poblaciones pre-históricas como históricas, como aquellas étnica y geográficamente diferentes. Este autor matiza que el tamaño del cráneo y el cuerpo no guardan correlación con el de los oscículos, lo que tiene un valor filogenético sustancial. Esto se debe traducir en muy pocas variaciones anatómicas para estas estructuras óseas. Quam \& Rak (2008) no encontraron diferencias morfológicas significativas salvo algunas diferencias morfométricas sutiles entre oscículos de hombre moderno y Neandertal que asocian a la evolución. Estos autores encontraron medidas que confirman sutiles variaciones con otras épocas del género Homo (en paréntesis nuestros resultados): largo total del manubrio de 8,01 mm DE 0,54 (8,18 mm DE 0,24); longitud del manubrio de 4,49 mm DE 0,29 (4,91 mm DE 0,25), y la proporción en porcentaje que ocupa la distancia de cabeza a proceso lateral (largo del manubrio) con el total de la distancia entre cabeza a ápice de manubrio (largo del manubrio) de 55,5\% DE 3,8 (60,11\% DE 3,47).

Unur (1993) reportó una variación del proceso anterior (como largo) en muestras de recién nacidos que no registramos en oscículos de adultos con medidas en promedio de 0,59 mm DE 0,21. Este autor señaló que este hallazgo como probable en recién nacidos, quizás pueda ser explicado al ser conformado embriológicamente de manera diferente al martillo, que lo hace por una ruta endocondral, mientras que el proceso anterior del martillo lo logra por osificación intramembranosa, a partir de células mesenquimales (os goniale) (Rodriguez Vazquez et al., 1993).

El registro de todas estas medidas óseas buscó corroborar y aportar a lo encontrado en otras investigaciones. De esta forma se amplía la información desde una perspectiva étnica y geográfica diferente, ya que no hay trabajos parecidos en población mestiza Colombiana. Finalmente, un análisis pareado de estas medidas encontró que no hubo diferencia de lado en los registros.

En términos generales encontramos que la morfología del martillo es muy homogénea y estable. En las muestras pareadas no encontramos diferencias significativas de lado.

RAMIREZ, L. M. \& BALLESTEROS, L. E. Anthropometry of the malleus in humans; A direct anatomic study. Int. J. Morphol., 31(1):177-183, 2013

SUMMARY: Morphometric knowledge of middle ear structures, including each of the oscicles, is important for the understanding of its biomechanics complexity. The structural and dimensional characteristics of the malleus are registered in 23 samples of Colombian adult mestizo population. Registered measures involve the area of the head, neck, the manubrium, the anterior and lateral process and the area of the incudo-maleolar joint. Three of nineteen records taken in this study could be carefully compared to other studies, which showed similarities. The total length of the malleus was $8,18 \mathrm{~mm} \mathrm{SD} 0.24$, the length of the handle was $4.91 \mathrm{~mm}$ SD 0.25 and the ratio of the length of the manubrium and the malleus total length was $60,11 \%$ of 3.47 . In accordance with the studied population, individual or paired variations were not found in the anatomy of the malleus that makes it a morphometrically uniform and stable bone.

KEY WORDS: Oscicles; Malleus; Manubrium; Anthropometry; Anatomy. 


\section{REFERENCIAS BIBLIOGRÁFICAS}

Anson, B. J. \& Donaldson, J. A. Surgical anatomy of the temporal bone. $3^{\text {ra }}$ ed. Philadelphia WB Saunders, 1991.

Arensburg, B., Harell, M., Nathan, H. The human middle ear ossicles, morphometry and taxonomic implications. J. Hum. Evol., 10:199-205,1981.

Aycan, K.; Unur, E. \& Bozkır, M.G. Anatomical study of malleus. J. Health Sciences., 1:152-58, 1990.

Bouchet, A., Giraud, M. Contrubituon a l'etude morphologique et radiologique des osselets de l'ouie. Compte rendu de l' Association des Anatomists 53 Congrés., 141:588-600, 1968.

Bruintje, T. D. The auditory ossicles in human skeletal remains from a leper cemetery in Chichester, England. $J$. Archaeological Science, 17:627-33, 1990.

Cole, F.G. History of comparative anatomy. London, Macmillan \& Co. Ltd., 69-120, 1949.

Crevecoeur, I. New discovery of an Upper Paleolithic auditory ossicle: the right malleus of Nazlet Khater 2. J. Hum. Evol., 52:341-5, 2007

Farahani, R. M. \& Nooranipour, M. Anatomy and anthropometry of human stapes. Am. J. Otolaryngol., 29:42-7, 2008.

Flohr, S.; Leckelt, J.; Kierdorf, U. \& Kierdorf, H. How reproducibly can human ear ossicles be measured? A study of inter-observer error. Anat. Rec., 293:2094-106, 2010.

Guyton \& Hall. Tratado de Fisiología Médica $11^{\circ}$ Ed. Elsevier España S.A., 2007.

Harada, O. \& Ishii, H. The Condition of the auditory ossicles in microtia. Plast. Reconst. Surg., 50:48-53, 1972.

Harneja, N. K. \& Chaturvedi, R. P. A study of the human ear ossicles. Indian J. Otolaryng. Head \& Neck Surg., 25:154-60, 1973.

Ishimoto, S.; Ito, K.; Kondo, K.; Yamasoba, T. \& Kaga, K. The role of the external auditory canal in the development of the malleal manubrium in humans. Arch. Otolaryngol. Head Neck Surg., 130:913-6, 2004.

Masali, M. The ear bones and the vertebral column as indications of taxonomic and postural distinctions among old world primates with reference to the origin of man. Rosenberg and Sellier, Torino, 1968.

Morris, D. P.; Bance, M.; van Wijhe, R.G.; Kiefte, M. \& Smith, R. Optimum tension for partial ossicular replacement prosthesis reconstruction in the human middle ear. Laryngoscope, 114:305-8, 2004.
Murugasu, E.; Puria, S. \& Roberson, J.B. Malleus-to-footplate versus malleus-to-stapes-head ossicular reconstruction prostheses: temporal bone pressure gain measurements and clinical audiological data. Otol. Neurotol. 26:572-82, 2005.

Olsewski, J. The morphometry of the ear ossicles in humans during development. Anat. Anz., 171:187-91, 1990.

Quam, R. \& Rak, Y. Auditory ossicles from southwest Asian Mousterian sites. J. Hum. Evol., 54:414-33, 2008.

Ramirez, L. M.; Ballesteros, L. E. Human Oscicular Chain Articulations: Asymmetric Sound Transmission. Int. J. Morphol., 28:1059-68, 2010

Rodriguez-Vazquez, J. F.; Merida, V. Jr. \& Jimenez, C. J. Relationships between the temporomandibular joint and the middle ear in human fetuses. J. Dent. Res., 72:62-6, 1993.

Sarrat, R.; Torres, A.; Guzman, A.G.; Lostalé, F. \& Whyte, J. Functional structure of human auditory ossicles. Acta Anat., 144:189-95,1992.

22. Sun, Q.; Gan, R.Z.; Chang, K.H. \& Dormer, K.J. Computerintegrated finite element modeling of human middle ear. Biomech. Model Mechanobiol., 1:109-22, 2002.

Tonndorf, J. \& Khanna, S. M. Tympanic-membrane vibrations in human cadaver ears studied by time-averaged holography. $J$. Acoust. Soc. Am., 52:1221-33, 1972.

Unur, E.; Aycan, K. \& Ekinci, N. The study of incus from morphometric view. Erciyes Medical J., 15:16-9, 1993.

Whyte, J.; Cisneros, A. I.; Urieta, J. J.; Yus, C.; Gañet, J.; Torres, A. \& Sarrat, R. Ontogenic peculiarities of the human tympanic ossicular chain. Acta Otorrinolaringol. Esp., 54:1-10, 2003.

\section{Dirección para correspondencia: Luis Miguel Ramirez \\ Grupo Estomatología Biomédica \\ Universidad de Antioquia \\ COLOMBIA}

Email: Imra3@yahoo.com

Recibido : 28-01-2012

Aceptado: 07-11-2012 